\title{
Molekulare Mechanismen der kutanen neurogenen Entzündung und des Juckreizes
}

\section{Molecular Mechanisms of Cutaneous Neurogenic Inflammation and Pruritus}

\author{
Autor \\ M. Steinhoff \\ Institut \\ Klinik und Poliklinik für Hautkrankheiten, Universitätsklinikum Münster
}

\section{Bibliografie}

DOI $10.1055 / \mathrm{s}-2006-944837$

Akt Dermatol 2006; 32;

463-467 @ Georg Thieme

Verlag KG Stuttgart · New York ISSN 0340-2541

Korrespondenzadresse

Univ.-Prof. Dr. med. Dr. rer. physiol. Martin Steinhoff

Klinik und Poliklinik für

Hautkrankheiten

Universitätsklinikum Münster

Von-Esmarch-Str. 58

48149 Münster

msteinho@uni-muenster.de

\section{Zusammenfassung \\ $\nabla$}

Untersuchungen der letzten Jahre werfen ein neues Licht auf die Rolle kutaner peripherer Nerven und etablierten ein modernes Konzept zum Verständnis Hauthomöostase, kutaner Entzündungen, Schmerz oder Juckreiz. Demnach existieren multiple bidirektionale Wege, bei denen das zentrale und periphere Nervensystem, das endokrine System, das Immunsystem und zahlreiche Hautzellen involviert sind. Über die involvierten Faktoren bei diesen immunmodulatorischen Mechanismen tauchen immer mehr Informationen auf, die zeigen wie Neuropeptide, Neurotransmitter, Neutrophine und Zytokine mit dem peripheren Nervensystem kommunizieren. Die Interaktion zwischen peripheren Nerven und dem Immunsystem wird durch verschiedene Typen kutaner Nervenfasern vermittelt, die diese Neuro-

Anatomie des peripheren sensorischen Nervensystems

$\nabla$

Vor einem Jahrhundert konnte in Tierexperimenten eine kutane Vasodilatation nach Durchtrennung dorsaler Nervenwurzeln ausgelöst werden, was den Grundstein zum Verständnis des Zusammenhanges zwischen dem Nervensystem und der Gefäßregulation (klassische neurogene Entzündung) legte. Später wurden nicht-mechanisch induzierbare polymodale hitze- und chemosensitive „kleine“ Nervenfasern (C- und A $\delta$ Nozizeptoren) identifiziert und charakterisiert, die erklärten, wie Nerven an der kutanen Entzündung beteiligt sind (reviewed in [1,2]). Jahrzehnte später konnte beobachtet werden, wie Erytheme und Überwärmung durch eine vorherige Behandlung der Haut mit mehrfach topisch ange-

* Preisträger der Berliner Stiftung für Dermatologie mediatoren freisetzen und spezifische Rezeptoren auf Keratinozyten, Mastzellen, Langerhanszellen, mikrovaskulären Endothelzellen, Fibroblasten und infiltrierenden Immunzellen aktivieren. Diese Interaktionen beeinflussen eine Vielzahl physiologischer und pathophysiologischer Vorgänge einschließlich der Organentwicklung, Differenzierung, Immunität, Vasoregulation, Rekrutierung von Leukozyten, Juckreiz und Wundheilung. Dieser Artikel beleuchtet neuere Erkenntnisse der neurokutanen Biologie und diskutiert die Rolle von Nerven für die Vermittlung von Hauterkrankungen wie Entzündungen, Brennen oder Juckreiz. Das Verständnis der Mechanismen und der Faktoren wie Neuromediatoren Vorgänge in der Haut kontrollieren, könnte zur Identifizierung neuer therapeutischer Strategien und somit Behandlung kutaner Entzündungen oder Juckreiz führen. wandtem Capsaicin über mehrere Tage inhibiert werden können. Nach dieser Behandlung waren die sensorischen Nerven „desensitisiert“. Über parakrine, juxtakrine oder endokrine Wege finden die freigesetzten Neuropeptide ihre Zielzellen und verursachen dort Rötung, Ödeme, Hyperthermie und Pruritus (bzw. Schmerz, Brennen, Brennschmerz). Aufgrund ihrer engen anatomischen Assoziation mit kutanen Nerven, scheinen Mastzellen und ihre freigesetzten Produkte eine wichtige Rolle für die Vermittlung neuronaler antidromer Antworten in der Haut zu spielen ( $\bullet$ Abb. 1). Die genauen molekularen Mechanismen und entscheidenden Rezeptoren für die verschiedenen biologischen Vorgänge variieren jedoch und bleiben weiterhin zu klären (wie z.B. die Rolle des Histamins und neuerer Histaminrezeptoren (z. B. Histamin-4-Rezeptor) bei der Immunantwort oder Juckreiz, die Bedeutung von Tryptase und Tryptaserezeptoren beim Menschen oder die Bedeutung von Prostaglandinen 


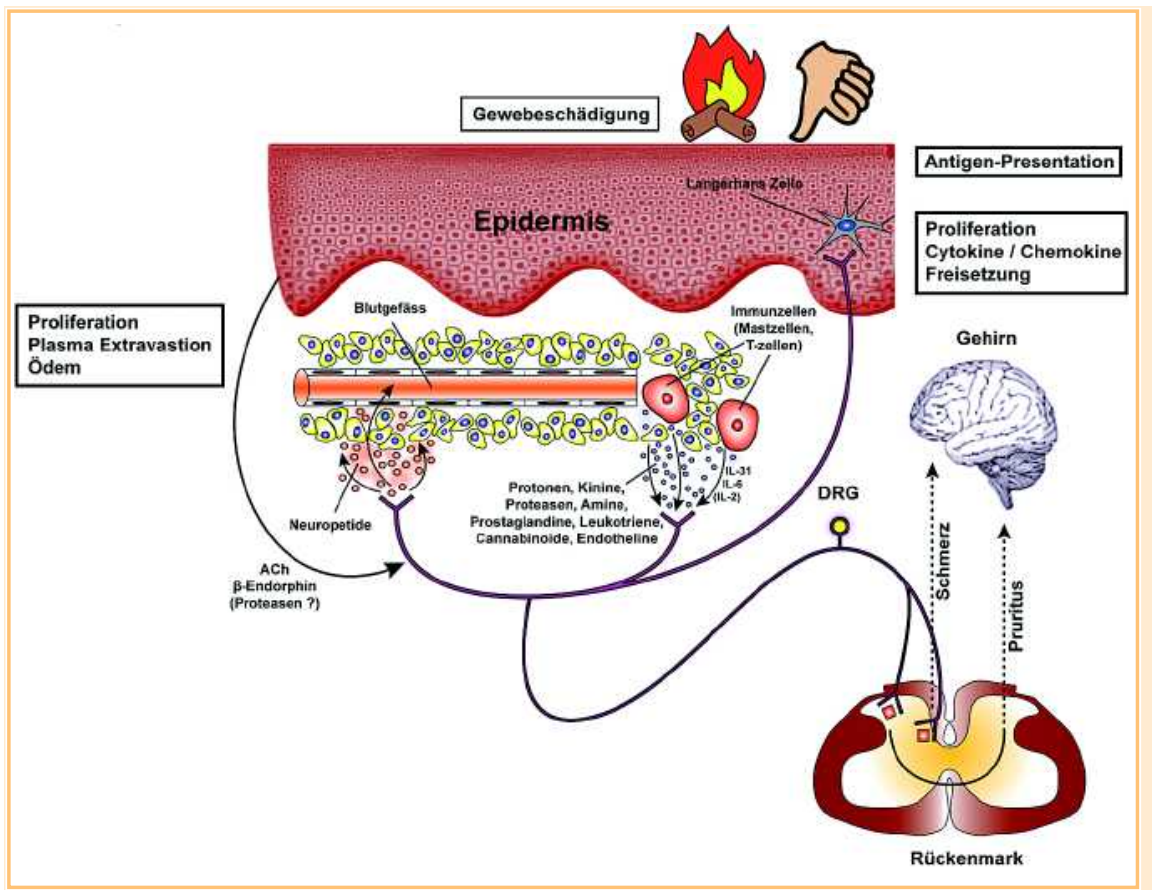

Abb. 1 Bedeutung des peripheren, sensorischen Nervensystems in der Haut.

Von der Umwelt auf die Haut treffende exogene (Trauma, Infektion, Noxen, Allergene, UV-Licht) oder endogen durch Entzündung oder „Stress“ (von ZNS oder Nebenniere freigesetzte Mediatoren) freigesetzte Botenstoffe (Mediatoren) stimulieren spezifische Untertypen von Rezeptoren (z. B. für Histamin, Prostaglandine, Cannabinoide, Proteasen, pH-Änderungen, Hitze, Kälte) auf peripheren Nervenendigungen von primär afferenten Neuronen („Schmerz- oder Juckreiz-Fasern“) (Schlüssel-Schloss-Prinzip). Mediatoren können z. B. von Keratinozyten (z. B. $\beta$-Endorphin), Endothelzellen, Mastzellen (Tryptase, Histamin, TNFa, Leukotriene) oder T-Lymphozyten (Interleukin-31) stammen. Zu beachten ist, dass einige Mediatoren die Aktivierung der Nerven stimulieren (Verstärkung von Juckreiz, Schmerz), andere abschwächen (Abschwächung). Die Nervenfasern übermitteln die Stimulation (Schmerz, Brennen, Juckreiz) mittels chemischer und elektrischer Signalleitung von der Haut zum Rückenmark. Innerhalb des Rückenmarks können
Schmerz- oder Juckreiz-Signale moduliert (verstärkt, abgeschwächt) werden (zentrale Sensibilisierung bzw. Desensibilisierung). Nach Überkreuzung des Rückenmarks werden die Signale moduliert zum Zentralnervensystem (Gehirn) geleitet. Die Aktivierung bestimmter Areale innerhalb des ZNS resultiert in der Wahrnehmung von Juckreiz oder Schmerz, was im Falle z. B. des Juckreizes zu „Unbehagen“ und somit zu einer „Kratz-Antwort“ (Kratzen) führt. Der in der Haut zusätzlich peripher vorhandene „Axon-Reflex" (Stimulation benachbarter peripherer Nervenfasern) kann sekundär zur Ausschüttung von Entzündungs-Botenstoffen (z. B. Substanz P, CGRP) führen, was zu den typischen Charakteristika einer akuten Entzündung (Schwellung, Rötung, Schmerzen) und ggf. Verstärkung des Juckreizes führt. Diese Abbildung berücksichtigt nicht die Interaktion zwischen Schmerz- und Juckreizfasern innerhalb des Rückenmarks. und Leukotrienen für die Aktivierung neuronaler Zellen bei verschiedenen Erkrankungen).

Primär afferente Neuronen exprimieren spezifische Rezeptoren für Kinine, Prostaglandine, Histamin, Tryptase, Vanilloide und Zytokine. Dies deutet darauf hin, dass ein interaktives Kommunikationsnetzwerk zwischen sensorischen Nerven und Immunzellen während der kutanen Inflammation existiert. Schließlich erzeugen die meisten Zellen, die Neuropeptidrezeptoren exprimieren, auch Neuropeptid-degradierende Peptide, wie die neutrale Endopeptidase (NEP) oder Angiotensin-converting enzyme (ACE), wodurch der durch die Neuropeptide verursachte inflammatorische Stimulus beendet wird. Entsprechend bilden Zellen, wie z.B. Keratinozyten und Makrophagen, die Rezeptoren für Neurotransmitter wie Acetylcholin und Noradrenalin synthetisieren, auch Enzyme, die die Effekte dieser Moleküle kontrollieren [3]. Auf diese Weise ist eine enge Interaktion zwischen Neuromediatoren, Zielzellrezeptoren und Neuropeptid-degradierenden Enzymen entscheidend für die Kontrolle der neurokutanen Kommunikation und der kutanen neurogenen Entzündung (๑ Abb. 1).

\section{Neuropeptide \\ $\nabla$}

Neuropeptide gehören zu einer Gruppe kleiner Peptide mit vier bis mehr als 40 Aminosäuren. Bislang konnten mehr als 20 Neuropeptide in der Haut identifiziert und bei unterschiedlichen Spezies charakterisiert werden. Bei der Bewertung der Funktion eines Neuropeptids müssen Organ- und auch Spezies-spezifische Effekte bei der Auswertung berücksichtigt werden. Die bekanntesten Neuropeptide in der Haut sind Substanz P (SP), Neurokinin (NK) A, Neurotensin, CGRP, VIP, Pituitary adenylate cyclase activating peptide (PACAP), Neuropeptid Y, Somatostatin (SOM), $\beta$-Endorphin, Enkephalin, Galanin, Dynorphin, ANP, $\alpha-$ oder $\gamma$-Melanozyten-stimulierendes Hormon (MSH), parathyroid Hormon-verwandtes Protein (PTHrP), Corticotropin-freisetzendes Hormon ( $\mathrm{CRH}$ ) oder Urocortin. Unter physiologischen oder pathophysiologischen Bedingungen (Trauma, Temperaturunterschiede, Entzündung, Juckreiz) sind kutane Zellen, wie Keratinozyten, mikrovaskuläre Endothelzellen, Merkelzellen, Fibroblasten und Leukozyten in der Lage einige der oben genannten Neuropeptide freizusetzen ( $\triangle$ Abb. 2 ) (Übersicht in $[1,4,5]$ ).

Als Beispiel, die Neuropeptide SP, NK-A und NK-B beeinflussen die Funktion von Keratinozyten, Endothelzellen, Mastzellen und Fibroblasten. Sie binden mit unterschiedlichen Affinitäten an die drei potenziellen Neurokininrezeptoren. Keratinozyten, 


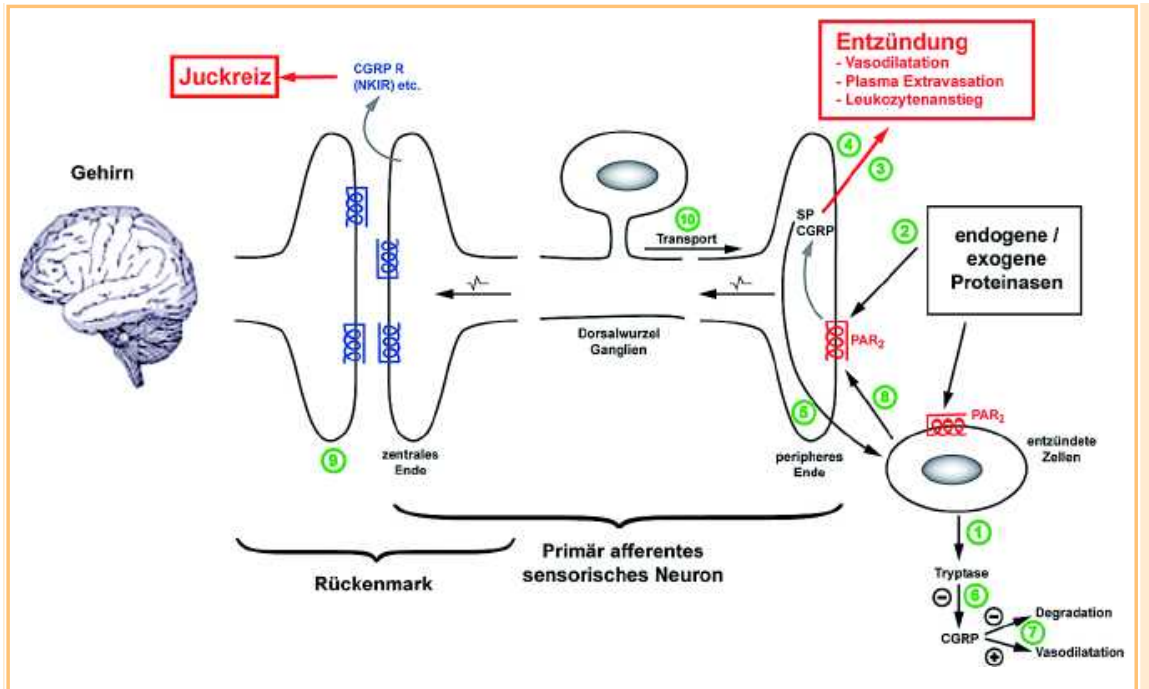

Abb. 2 Protease-aktivierte Rezeptoren (PARs) spielen eine Schlüsselrolle bei Entzündung und Juckreiz.

1. Von degranulierten Mastzellen ausgeschüttete Mastzell-Tryptase aktiviert den Rezeptor PAR2 an der Plasmamembran von sensorischen Nervenendigungen. 2. Die Aktivierung von PAR2 durch Tryptase, Trypsin, verschiedene Kallikreinen und vermutlich exogene Proteasen (Bakterien, Hausstaubmilben) stimuliert die Ausschüttung von CGRP und der Tachykinine SP und Neurokinin A (NKA) an sensorischen Nervenendigungen. 3. CGRP interagiert mit dem CGRP1-Rezeptor, und induziert so eine arterielle Gefäßerweiterung (Rötung, Erwärmung). 4. SP interagiert mit dem entsprechenden Rezeptor (NK1R) auf venösen Blutgefäßzellen (Endothelzellen), um dort eine Ödembildung (Schwellung) auszulösen. 5. Substanz P stimuliert die Aktivierung von Mastzellen, was zur verstärkten Entzündungsreaktion oder Juckreiz führt. 6. Mastzell-Tryptase baut aber auch Neuropeptide wie CGRP ab und terminiert so dessen Effekt. 7. CGRP hemmt auch die SP Degradation durch Aktivierung von Peptidasen wie neutrale Endopeptidase (NEP) und erhöht so zusätzlich die SP-Ausschüttung, wodurch der entzündliche Effekt verstärkt wird. 8. Mediatoren von Mastzellen und anderen Entzündungszellen stimulieren auch die Ausschüttung von Neuropeptiden von Nervenendigungen. 9. Auf der Ebene des Rückenmarks führt die PAR2 induzierte intrazellulare Ca-Mobilisierung zur Ausschüttung von CGRP und SP an zentralen Nervenenden, wodurch Rezeptoren aktiviert werden, die die Juckreiz- oder Schmerz-Reaktion zum zentralen Nervensystem leiten. 10. Während einer Entzündung kann PAR2 von den Neuronen zum Nervenende transportiert werden, was zur Erhöhung der Rezeptordichte und so zu verstärkter Stimulierbarkeit führt.
Langerhanszellen, Merkelzellen, Fibroblasten, Mastzellen und Endothelzellen exprimieren funktionelle Neurokininrezeptoren, während die G Proteine von Mastzellen zusätzlich durch SP in einem Nicht-Rezeptor-vermittelten Weg (durch direkte Aktivierung von G Proteinen) aktiviert werden können. Ob eine Rezeptor-Fehlregulation oder die Beendigung der Rezeptoraktivität (z.B. durch Polymorphismen und/oder Störungen in der intrazellulären Signaltransduktion) aktiv zu kutanen Erkrankungen beitragen, ist gegenwärtig unbekannt. NK-1R Gen-defiziente Mäuse zum Beispiel bilden unter normalen Bedingungen keine spezifischen Haut-verwandten Phänotypen aus, aber sie zeigen dafür charakteristische Anzeichen von Ödemen und Plasmaextravasation. In einem experimentell induzierten Wundheilungsmodell konnten keine NK-1R vermittelten Effekte beobachtet werden. Darüber hinaus belegen Studien, dass Neurokininrezeptor-Antagonisten aussichtsreiche Kandidaten zum Beispiel zur Behandlung von Entzündung, Schmerz oder Juckreiz sein können (Übersicht in [1]).

\section{Klassische Neurotransmitter}

Acetylcholin, Adrenalin und Noradrenalin sind kleine, nicht aus Peptiden bestehende Botenstoffe, die von Neuronen und nichtneuronalen Zellen in verschiedensten Geweben, einschließlich der Haut, produziert werden. Ursprünglich wurden diese Moleküle als wichtige Mediatoren beschrieben, die in autonomen Nervenfasern freigesetzt werden. Neuere Studien belegen, dass adrenerge und cholinerge Transmitter eine wichtige Rolle in der kutanen Homöostasie und Entzündung spielen. Zum Beispiel können Catecholamine die Kapazität der Antigenpräsentation in murinen Langerhanszellen über die Aktivierung des $\beta_{2}$-adrenergen Rezeptors auf diesen Zellen herunter regulieren. Adrenalin inhibiert ebenfalls das Auslösen einer Kontakthypersensibilisierung nach epikutaner Verabreichung von Haptenen in vivo, was eine direkte Rolle der autonomen Nerven in der kutanen Immunmodulation nahe legt. Acetylcholin spielt ebenfalls eine wichtige Funktion für die Regulation von Makrophagen, z.B. durch Herunterregulation von NF-kB und Verminderung der Freisetzung von TNF-a [3].

\section{Neurotrophine in der Haut}

$\nabla$

Die Säugerhaut exprimiert eine Vielzahl von neurotrophen Wachstumsfaktoren, wie z.B. den Nervenwachstumsfaktor (NGF), Neurotrophin-3 und Neurotrophin-4/5, oder brain-derived neurotrophic factor (BDNF), die essentiell sind für Wachstum, Proliferation und Aufrechterhaltung von Nerven. Kutane Neurotrophine werden durch sensorische und sympathische Neuronen und nicht neuronale Zellen exprimiert, wodurch verschiedene biologische Modalitäten wie Nozizeption, Propriozeption, Mechanorezeption, Nervenwachstum und Entwicklung, Apoptosis, epidermale Homöostasie, Inflammation, Haarwachstum und Melanogenesis reguliert werden. Verschiedene Beobachtungen deuten darauf hin, dass Neurotrophine sich an dem neuroimmunologischen Netzwerk beteiligen (z.B. die Gen-Expression von NGF und Neutrophin 3, 4 und 5 können durch Zytokine wie IL-6 und löslichem IL-6 Rezeptor moduliert werden) (Übersicht in $[1,2,4]$ ). 


\section{Rolle von Hitze- und Kälterezeptoren in der Haut $\nabla$}

Topisch angewendetes Capsaicin, ein Vanillylalkaloid aus der roten scharfen Chilischote, ruft durch die selektive Aktivierung kleiner sensorischer Nervenfasern und der Auslösung einer neurogenen Entzündung einen brennenden Schmerz hervor. Die Langzeitanwendung von Capsaicin führt darüber hinaus wahrscheinlich durch einen neurotoxischen Effekt in sensorischen Nerven und evtl. durch intrazellulär vermittelte Desensitisierung zur Termination der entzündlichen Reaktion. Dieser Effekt wird in einigen Ländern therapeutisch zur Behandlung chronischer Krankheiten mit Schmerz (Gelenkrheuma) oder Juckreiz (Prurigo) genutzt. Aufgrund seiner Nebenwirkungen (Induktion, Verstärkung einer Entzündung) ist es für den Einsatz inflammatorischer Erkrankungen daher weniger geeignet. Zukünftig wünschenswert wären daher Substanzen, die den Capsaicinrezeptor TRPV1 zwar desensitisieren (inaktivieren), ohne jedoch zunächst eine neurogene Entzündung mit Schmerz durch Neuropeptidfreisetzung hervorzurufen.

Transiente Rezeptorpotenziale (TRPs) sind eine neuartige Familie der temperatur-sensitiven Rezeptoren mit sechs Transmembrandomänen. TRPs sind nicht selektive Kationenkanäle, die mit anderen Mitgliedern nicht-selektiver Kalziumkanäle strukturell verwandt sind. Caterina et al. [6] konnten kürzlich die erfolgreiche Klonierung des Ratten-Capsaicinrezeptors (TRP Vanilloid Rezeptor 1 [TRPV1]) beschreiben. Ein niedriger pH-Wert, der eine inflammatorische Reaktion begleitet, kann die Reaktion des TRPV1 auf schädliche Stimuli erhöhen, was darauf hindeutet, dass die Antwort der sensorischen Nervenfasern während einer neurogenen Entzündung erfolgt und - zumindest teilweise von der Aktivierung des TRPV1 durch einen Überschuss von Protonen, endogenen Canabinoiden und möglicherweise Bradykinin hervorgerufen wird. Neuere Daten unterstützen eine direkte Rolle von TRPV1 bei der neurogenen Entzündung ( $\bullet$ Abb. 1): Trotz allem ist die präzise Rolle der verschiedenen TRPs (TRPV1, TRPV2, TRPV3, TRPV4, TRPM8, TRPA1) bei Hauterkrankungen noch unklar. Vorstellbar wäre z. B. eine Rolle in der Pathophysiologie der Kälte- oder Wärmeurticaria oder Rosazea (Übersicht in [7]).

\section{Bedeutung Proteinase-aktivierter Rezeptoren (PARs) fuir die kutane neurogene Entziindung und Juckreiz $\nabla$}

Neuere Daten deuten stark darauf hin, dass Serinproteasen wie Thrombin, Cathepsin G, Tryptase und Trypsin nicht nur Enzyme sind, die Proteine oder Peptide im extrazellulären Raum degradieren, sondern zusätzlich während einer Inflammation und einer Immunantwort wichtige Effekte wie die Zytokinfreisetzung, Zellmigration, Rekrutierung von Leukozyten und Aktivierung von Endothelzellen vermitteln [1].

Diese Prozesse werden zuletzt durch die Spaltung und Aktivierung von Proteinase-aktivierten Rezeptoren (PARs) vermittelt. PARs sind G Protein-gekoppelte Rezeptoren mit sieben transmembranären Domänen, die durch proteolytische Spaltung aktiviert werden. Bis jetzt konnten 4 PARs kloniert und charakteri- siert werden. Die Aktivierung von PAR Rezeptoren führt zur Auslösung von Signaltransduktionskaskaden, die unmittelbar an Entzündungsvorgängen, Schmerz oder Juckreiz verbunden sind († Abb. 2) (Übersicht in [8]).

Jüngste Daten weisen darauf hin, dass PAR-1 und PAR-2 bei der neurogenen Entzündung involviert sind [9]. Dem liegt der Mechanismus zugrunde, dass Proteasen auf sensorischen Neuronen PAR-2 aktivieren, und so Neuropeptide freisetzen, die wiederum Gefäßerweiterung und Plasmaextravasation (Ödembildung) hervorrufen. Der Ligand von PAR-2, Mastzell-Tryptase, induziert auch die Infiltration des Entzündungsgebietes mit neutrophilen Granulozyten und stimuliert die Zytokinsekretion. Bei verschieden Entzündungen der Haut können Tryptase-freisetzende Mastzellen in enger Nähe zu PAR-2 exprimierenden Zellen wie Keratinozyten und Endothelzellen oder Nerven gefunden werden. Vor kurzem durchgeführte Studien an PAR-2 defizienten Mäusen zeigten, dass PAR-2 eine wichtige Rolle bei der Kontaktdermatitis in vivo spielt.

\section{Kontrolle der Neuropeptidrezeptor-Aktivierung durch Neuropeptid-degradierende Enzyme $\nabla$}

Neuere Studien deuten an, dass NEP (Neutrale Endopeptidase), ACE (Angiotensin-converting enzyme) oder ECE (Endothelinconverting enzyme) eine wichtige Bedeutung für die Kontrolle der neurogenen Mechanismen in der Haut spielen. Während ACE fähig ist, die Tachykinine SP, Bradykinin und Angiotensin zu degradieren, spaltet NEP zusätzlich NK-A, NK-B, VIP, PACAP, atriales natriuretisches Peptid und Endotheline. NEP und ACE konnten beide in vaskulären Endothelzellen, Hautfibroblasten und Keratinozyten identifiziert werden. ACE wird überwiegend auf der luminalen Seite des vaskulären Endothels gefunden, was auf eine hauptsächliche Bedeutung für die vaskuläre Antwort (Vasodilatation, Plasmaextravasation und Leukozyten-Endothel Interaktion) hinweist.

In-vivo-Studien an $\mathrm{NEP}^{-/-}$(Gen-defizienten) Mäusen zeigten eine signifikante Zunahme der Plasmaextravsation und der kutanen Entzündung im Mausmodell der experimentell induzierten Kontaktdermatitis, was eine antiinflammatorische Rolle von NEP bei der kutanen Entzündung nahe legt. Bei der kutanen Entzündung (Kontakthypersensitivität) wurden für ACE ähnliche Effekte beobachtet [10,11].

Diese Befunde deuten an, dass die Hochregulation von NEP eine potenzielle therapeutische Behandlung zur Minderung der proinflammatorischen Effekte der neurogenen Entzündung darstellt. Die Herunterregulation von NEP und ACE könnte zu einer unkontrollierten Stimulation der Neuropeptide und zu einer chronischen Entzündung oder verstärktem Juckreiz führen.

\section{Neuronale Zytokinrezeptoren in der Haut} $\nabla$

Jüngere Untersuchungen belegen die Expression von Zytokinrezeptoren auf neuronalen Zellen von Mäusen und Menschen ( Abb. 1). Somit könnten Zytokine auch direkt über die Aktivierung neuronaler Zellen an der Regulation der neuro-immunologischen Achse in der Haut und auch pathophysiologischen Vorgängen wie Entzündung, Juckreiz oder Schmerz beteiligt sein. Kandidaten hierfür sind z. B. IL-2, IL-6 oder IL-31. IL-31, zum Beispiel, induziert Juckreiz und Entzündung in Mäusen [5,12,13]. 


\section{Zusammenfassung und Perspektiven \\ $\nabla$}

Zusammengefasst spielt die Interaktion der Haut mit dem peripheren sowie dem zentralen Nervensystem eine entscheidende Rolle für die physiologische Hautfunktion und -entwicklung, aber auch bei verschiedenen Erkrankungen wie z.B. Trauma, Wundheilung, atopische Dermatitis, Urticaria oder Rosazea. Vor kurzem gemachte Entdeckungen über die molekularen Mechanismen von Neuropeptiden und ihr Zusammenspiel mit Neuropeptidrezeptoren und Peptidasen zusammen mit der Entwicklung moderner molekularer und neurophysiologischer Techniken bieten spannende Einblicke in ein komplexes Netzwerk von Haut, Nerven und Immunsystem bei Entzündung, Schmerz und Juckreiz oder Brennen. Hierdurch sind neue therapeutische Strategien z.B. zur Behandlung von Erkrankungen wie atopischer Dermatitis, Rosazea, Urticaria oder von Symptomen wie Juckreiz oder Schmerz zu erwarten.

\section{Abstract}

\section{Molecular Mechanisms of Cutaneous Neurogenic Inflammation and Pruritus \\ $\nabla$}

Investigations during the last years shed a new light on the role of cutaneous peripheral nerves and established a modern concept in our understanding of skin homeostasis, inflammation, immunomodulation, pain and itch. Nerve communicate via multiple bidirectional pathways with the endocrine system, the immune system and numerous skin cells. Information emerges about the various factors involved in immunomodulation or inflammation such as neuropeptides, classical neurotransmitters, neutrophins, oxygen species or cytokines released by or communicating with the nervous system. The interaction between peripheral nerves and the immune system is mediated by various types of cutaneous nerve fibres, which release neuromediators and activate specific receptors on keratinocytes, mast cells, Langerhans cells, microvascular endothelial cells, fibroblasts or infiltrating immune cells. These interactions affect a multitude of physiological and pathophysiological processes including organ development, differentiation, immunity, vasoregulation, recruitment of leukocytes, itch or wound healing. This article illuminates recent insights of the neurocutaneous network and discusses the role of nerves for the mediation of skin diseases such as inflammation, immunity, burning or itch. Understanding the mechanisms and factors of how neuromediators control processes in the skin will lead to the identification of new therapeutic strategies and thus treatment of various diseases or itch.

\section{Literatur}

1 Roosterman D, Goerge T, Schneider SW, Bunnett NW, Steinhoff M. Neuronal Control of Skin Function: The Skin as a Neuroimmunological Organ. Physiol Rev 2006; (in press)

2 Steinhoff M, Stander S, Seeliger S, Ansel JC, Schmelz M, Luger T. Modern aspects of cutaneous neurogenic inflammation. Arch Dermatol 2003; 139: $1479-1488$

3 Czura CJ, Friedman SG, Tracey KJ. Neural inhibition of inflammation: the cholinergic anti-inflammatory pathway. J Endotoxin Res 2003; 9: 409-413

4 Slominski A, Wortsman J, Luger T, Paus R, Solomon S. Corticotropin releasing hormone and proopiomelanocortin involvement in the cutaneous response to stress. Physiol Rev 2000; 80: 979-1020

5 Sun $R Q$ Tu YJ, Lawand NB, Yan JY, Lin Q Willis WD. Calcitonin gene-related peptide receptor activation produces PKA- and PKC-dependent mechanical hyperalgesia and central sensitization. J Neurophysiol 2004; 92: 2859-2866

6 Caterina MJ. Vanilloid receptors take a TRP beyond the sensory afferent. Pain 2003; 105: 5-9

7 Paus R, Schmelz M, Biro T, Steinhoff $M$. Frontiers in pruritus research: scratching the brain for more effective itch therapy. J Clin Invest 2006; 116: $1174-1186$

8 Steinhoff M, Buddenkotte J, Shpacovitch V, Rattenholl A, Moormann C, Vergnolle $N$ et al. Proteinase-activated receptors: transducers of proteinase-mediated signaling in inflammation and immune response. Endocr Rev 2005; 26: 1-43

9 Steinhoff M, Vergnolle N, Young SH, Tognetto M, Amadesi S, Ennes HS et al. Agonists of proteinase-activated receptor 2 induce inflammation by a neurogenic mechanism. Nat Med 2000; 6: 151-158

10 Scholzen TE, Steinhoff M, Sindrilaru A, Schwarz A, Bunnett NW, Luger TA et al. Cutaneous allergic contact dermatitis responses are diminished in mice deficient in neurokinin 1 receptors and augmented by neurokinin 2 receptor blockage. Faseb J 2004; 18: 1007 - 1009

11 Scholzen TE, Sunderkotter C, Kalden DH, Brzoska T, Fastrich M, Fisbeck T et al. Alpha-melanocyte stimulating hormone prevents lipopolysaccharide-induced vasculitis by down-regulating endothelial cell adhesion molecule expression. Endocrinology 2003; 144: 360 - 370

12 Dillon SR, Sprecher C, Hammond A, Bilsborough J, Rosenfeld-Franklin M, Presnell SR et al. Interleukin 31, a cytokine produced by activated T cells, induces dermatitis in mice. Nat Immunol 2004; 5: 752 - 760

13 Homey B, Steinhoff M, Ruzicka T, Leung DY. Cytokines and chemokines orchestrate atopic skin inflammation. J Allergy Clin Immunol 2006; 118: $178-189$ 